\title{
Multifocal abscesses due to multiresistant Escherichia coli after transrectal ultrasound-guided prostate biopsy
}

Matthew J Roberts Resident Medical Officer ${ }^{1,2}$

Anisha Parambi MB BS, Resident Medical Officer

Lucinda Barrett MB BS, MRCP, Clinical Observer

Paul Hadway MB BS, FRCS Fellow' Robert A (Frank)
Gardine
AM, MB BS, MD, FRACS
Urologist,' and
Professor of Urology

Krispin M Hajkowicz MB BS, FRACP Infectious Diseases Physician $^{3}$

John Yaxley MB BS, FRACS Urologist $^{1}$

1Department of Urology, Royal Brisbane and Women's Hospital, Brisbane, QLD.

2 University of Queensland Centre for Clinical Research Brisbane, QLD.

3 Infectious Diseases Unit. Royal Brisbane and Women's Hospital, Brisbane, QLD.

john@

yaxley-urology.com

doi: 10.5694/mjal2.11719
We report an unusual case of multidrug-resistant Escherichia coli bacteraemia causing multifocal abscesses, septic arthritis, lumbar discitis and osteomyelitis after transrectal ultrasound-guided prostate biopsy, requiring restricted antibiotics and surgical debridement. This case highlights the importance of risk assessment, prophylactic and therapeutic antimicrobial use, and urology-infectious diseases collaboration to improve clinical outcomes after such procedures.

\section{Clinical record}

An asymptomatic 61-year-old white man with comorbid obesity and hypertension underwent a transrectal ultrasound-guided prostate biopsy (TRUSPB) after an abnormal digital rectal examination. His serum prostate-specific antigen (PSA) level was $3.4 \mathrm{ng} / \mathrm{mL}$ (reference interval, $0.3-$ $4.5 \mathrm{ng} / \mathrm{mL}$ ). As per local guidelines, empirical antimicrobial prophylaxis with trimethoprim $300 \mathrm{mg}$ daily was commenced 3 days before and continued for 4 days after the TRUSPB, as well as intravenous gentamicin $240 \mathrm{mg}$ after sedation. Histological examination of the biopsy sample showed prostate cancer (Gleason 3+4 in 6/18 cores), with no seminal vesicle sampling reported.

The patient returned the day after the TRUSPB with fevers. He was prescribed ampicillin and gentamicin on admission, after blood and urine samples were collected for culture. These specimens subsequently grew an Escherichia coli, which showed a multidrug-resistant (MDR) profile, but without extended-spectrum $\beta$-lactamase (ESBL) production. Antimicrobial susceptibility testing using the VITEK 2 system (bioMérieux) showed resistance to ampicillin, gentamicin, tobramycin, trimethoprim, sulfamethoxazole-trimethoprim and ciprofloxacin, and susceptibility to cephazolin, ceftriaxone, amikacin, nitrofurantoin and meropenem. Susceptibility to $\beta$-lactam- $\beta$-lactamase combinations was variable. Etest (bioMérieux) showed a minimum inhibitory concentration of $0.032 \mathrm{mg} / \mathrm{L}$ for ceftriaxone (susceptible) and $0.25 \mathrm{mg} / \mathrm{L}$ for fosfomycin (susceptible).

Further questioning found that the patient had a recent history of travel to South-East Asia and previous use of fluoroquinolone antibiotics. He was treated with intravenous cephazolin but his temperature continued to spike above $38^{\circ} \mathrm{C}$. Magnetic resonance imaging (MRI) of his pelvis revealed a large prostatic abscess (Box 1), which was drained by transurethral resection. Tissue culture was consistent with the MDR E. coli previously isolated.

Despite ongoing cephazolin therapy, the patient developed increasing pain over the right sternoclavicular joint.

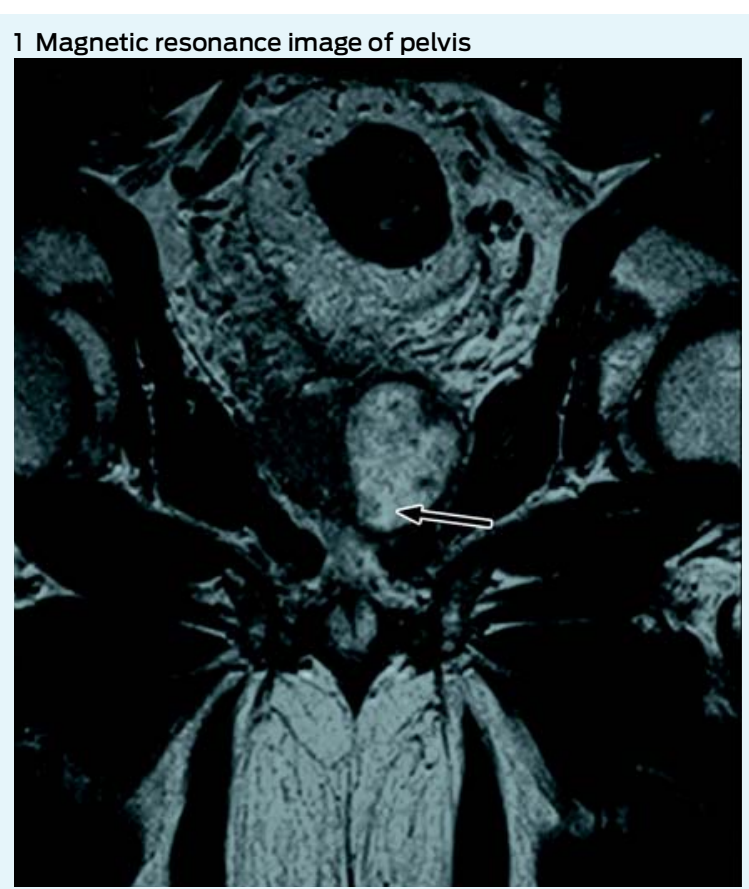

T1 sequence demonstrating a high T2 signal and heterogeneous appearance due to an abscess in the left lobe of the prostate $(2.2 \times 2.5 \times 3.7 \mathrm{~cm})($ arrow $)$, which extends into the prostatic apex.

Computed tomography imaging demonstrated changes consistent with a joint effusion, localised oedema, septic arthritis and osteomyelitis of the upper manubrium and medial right clavicle (Box 2). After initial conservative management without symptom resolution, surgical debridement was performed, and culture of the excised tissue grew MDR E. coli. The patient subsequently complained of lumbar back pain, and MRI of the lumbar spine showed changes consistent with an epidural abscess together with immediately adjacent discitis and osteomyelitis at the L4-5 vertebrae (Box 3). An orthopaedic consultation recom- 


\section{Computed tomography scan of chest}

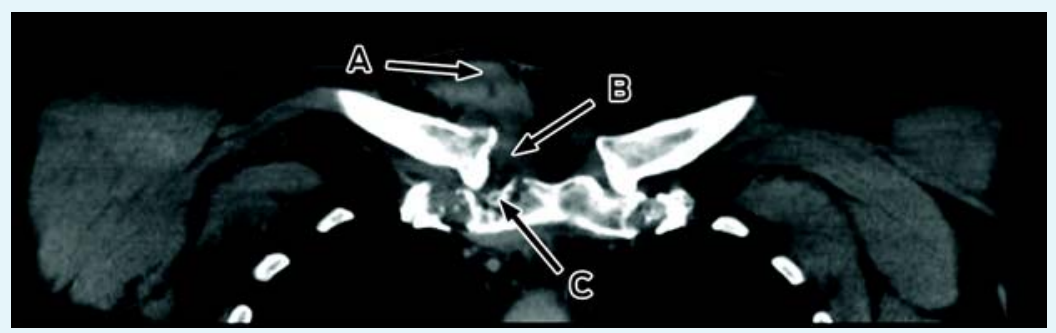

Scan demonstrating soft tissue oedema localised to the right sternoclavicular joint $(\boldsymbol{A})$ and appearances of an associated joint effusion ( $6 \mathrm{~cm}$ diameter) (B); and gas locules and demineralisation of the medial right clavicle, with lucency of the upper manubrium suggestive of septic arthritis and associated osteomyelitis (C).

\section{Magnetic resonance image of spine}

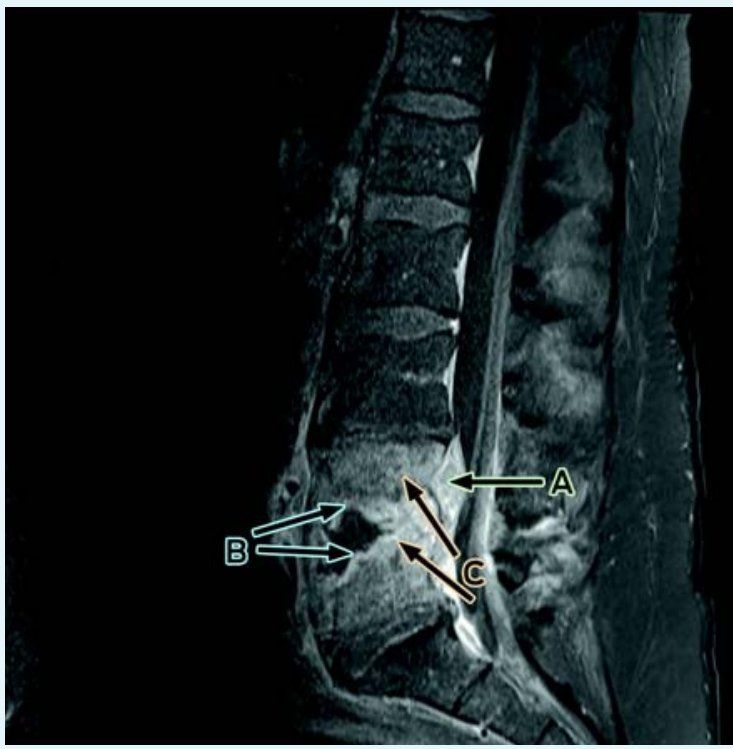

Tl fat-suppressed sequence demonstrating low signal collection posterior to the $L 4$ vertebra, consistent with an epidural abscess $(2.1 \times 1.3 \times 1.5 \mathrm{~cm})(\boldsymbol{A})$, and diffuse enhancement of the end plates and enhancement of the $L 4$ and $L 5$ vertebral bodies, consistent with discitis (B) and osteomyelitis (C).

mended conservative management, and the patient's primary care was transferred to infectious diseases physicians. After 5 weeks of intravenous cephazolin therapy in hospital, he was discharged with a treatment regimen of intravenous ceftriaxone $2 \mathrm{~g}$ daily delivered by an elastomeric infusion device (Baxter) managed by the home-based acute care service.

The patient was readmitted the day after discharge with increasing abdominal pain and continuing back pain. Repeat MRI of the lumbar spine showed slight radiological progression. The ceftriaxone dose was increased to $2 \mathrm{~g}$ twice daily, with ongoing conservative management of the spinal osteomyelitis. At the end of this second long-term admission (47 days), the patient had made significant clinical improvement and was discharged for ongoing outpatient-based rehabilitation, with a regimen of oral fosfomycin $3 \mathrm{~g}$ daily. In total, he received 10 weeks of intravenous antibiotics and 8 weeks of oral fosfomycin.
This case represents an unusual cascade of infectious complications - bacteraemia, septic arthritis, lumbar discitis, vertebral osteomyelitis and multifocal (prostatic and epidural) abscess formation - after a TRUSPB procedure. In isolation, these complications have been reported rarely, but we believe this series of infectious complications of TRUSPB is unique. The case serves as a timely reminder of the risks and possible consequences of prostate cancer detection, the difficulties in treating infectious complications in the era of gram-negative antimicrobial resistance, and the importance of collaboration between urology and infectious diseases physicians to optimise management.

A lack of consensus persists regarding the benefits of early detection of prostate cancer, including the PSA trigger point for recommending biopsy. The diagnostic process is commonly associated with periprocedural symptoms. Fortunately, most biopsy-related symptoms, such as haematuria and haematospermia, are benign and self-limiting. ${ }^{1}$ Infectious complications of TRUSPB are less common but are well documented, with the causative mechanism believed to be inoculation of the prostate, blood vessels and urine with bacterial flora from the rectal mucosa, and subsequent systemic dissemination. ${ }^{1,2}$ The most common manifestations are fever and genitourinary tract infection, while hospital admission and bacteraemia occur less frequently $(0.5 \%$ and $0.3 \%$ of all prostate biopsies performed, respectively). ${ }^{1}$ Urosepsis requiring intensive care unit (ICU) admission ( $0.08 \%$ of all biopsies) or causing death is rare. ${ }^{1}$ It is suspected that these rates may be higher in Australia than elsewhere, with a reported 1\% risk of bacteraemia and $0.2 \%$ risk of ICU admission likely to be underestimates. ${ }^{3}$

There has recently been concern that hospital admissions after TRUSPB may be rising, ${ }^{2}$ with a Canadian study reporting a threefold increase from $0.52 \%$ during 2002 2009 to $2.15 \%$ in $2010-2011 .^{1}$ Furthermore, at a total annual cost of around $£ 7.7-11.1$ (A\$11.4-16.5) million, the economic burden of post-TRUSPB infections in England and Wales has been estimated to be higher than that due to methicillin-resistant Staphylococcus aureus and Clostridium difficile infections. ${ }^{4}$ Although this cost may be an overestimation as it is based on an average hospital stay for gram-negative septicaemia of 14.2 days, ${ }^{4}$ while the median length of hospital stay for post-TRUSPB infections is 4 days, ${ }^{1}$ it adds to concerns regarding this issue.

$E$. coli is the pathogen most commonly responsible for post-TRUSPB infections. ${ }^{1}$ Bacterial mutations carrying genetic elements conferring antibiotic resistance and/or ESBL production are being increasingly reported, attributed to varying antimicrobial use by region. ${ }^{5,6}$ Fluoroquinolone resistance is increasingly common in isolates causing post-TRUSPB infection and is particularly problematic in Asian countries, specifically India, where fluoroquinolone resistance is reported in $>80 \%$ of clinical $E$. coli isolates. ${ }^{1,5,6}$ In some regions, this has been accompanied by the emergence of bacteria resistant to other broad-spectrum antimicrobial drugs, including carbapenems (3\% of clinical isolates tested for antimicrobial susceptibility in Hong Kong and Singapore). ${ }^{6}$ Our patient described previ- 
ous fluoroquinolone use and travel to South-East Asia in the weeks before the TRUSPB, both of which are identified as risk factors for fluoroquinolone resistance and postTRUSPB infection. ${ }^{5}$ Prior fluoroquinolone use results in a predominance of fluoroquinolone-resistant isolates in the rectum due to selection pressures, while overseas travel, particularly to South-East Asia, may favour acquisition of fluoroquinolone-resistant isolates as a result of contact with health care facilities or water sources contaminated with such bacteria. ${ }^{5}$ Other risk factors for post-TRUSPB infections include previous biopsy, immunosuppression (eg, diabetes, chronic obstructive pulmonary disease) and recent (past month) hospitalisation. ${ }^{1}$ None of these risk factors were present in our patient.

Antimicrobial prophylaxis has been shown to reduce infectious complications after TRUSPB. ${ }^{4}$ Oral fluoroquinolones are recommended as first-line antimicrobial prophylaxis by the American Urological Association, European Association of Urology and the Australian Therapeutic guidelines (specifically in Australia, ciprofloxacin $500 \mathrm{mg}$ orally, as a single dose, 1 hour before the procedure)., ${ }^{4,7}$ Second-generation fluoroquinolones are most frequently used because of their high oral bioavailability, broadspectrum efficacy against gut flora, and desirable prostatic tissue penetration, but they have a low resistance barrier to Enterobacteriaceae. The duration and frequency of antimicrobial prophylaxis varies across institutions, as does the addition of other antimicrobial agents, such as broadspectrum penicillins and aminoglycosides. Empirical antibiotic treatment for men presenting with post-TRUSPB infection should include a carbapenem if the patient has a history of travel to the Indian subcontinent or South-East Asia, is immunosuppressed or has severe sepsis. ${ }^{6}$

In our patient, fosfomycin monotherapy was successfully used as oral follow-on treatment after an extensive period of intravenous antibiotic use. This somewhat novel approach was based on literature showing both satisfactory bone and joint penetration of fosfomycin and clinical efficacy in bone and joint infections. ${ }^{8}$ Further prospective dose-ranging studies (which test different doses to determine the best or most suitable efficacy of a particular drug) of fosfomycin alone or in combination with other antibiotics for MDR gram-negative bacterial bone and joint infections are indicated. Although fosfomycin is an established antimicrobial agent in Europe, where it has had decades of clinical use, it is not currently approved by the Therapeutic Goods Administration and is not listed on the Australian Register of Therapeutic Goods. We accessed supplies from Germany through the Special Access Scheme.

Transperineal prostate biopsy has been reported to reduce the likelihood of biopsy-associated infections, but there are limitations to its adoption as a routine initial biopsy approach. ${ }^{4,9}$ These include logistical issues, as it has a longer procedure duration than TRUSPB, due to spinal or general anaesthesia requirements and use of a template- guided stereotactic method for needle placement. ${ }^{9}$ Transperineal prostate biopsy may also have a higher risk of acute urinary retention, requiring hospitalisation. ${ }^{9}$

Recently, in addition to advocacy for povidone-iodine rectal cleansing before biopsy, ${ }^{10}$ preprocedural rectal culturing on selective media to identify fluoroquinoloneresistant pathogens and predict infectious complications has been proposed. ${ }^{11}$ Rectal culturing and surveillance for ESBL-producers may also facilitate targeted antimicrobial prophylaxis, lower post-TRUSPB infection rates and reduce health care costs. ${ }^{10}$ However, this method is currently not widely available and remains experimental until advantages of its routine implementation are demonstrated clearly.

This case of infectious complications after TRUSPB highlights the benefits of close collaboration between urology and infectious disease physicians to inform risk assessment and antimicrobial prophylaxis before TRUSPB. It also emphasises the importance of early recognition and use of appropriate broad-spectrum antimicrobial agents to minimise adverse outcomes in these patients.

Acknowledgements: We thank Dr Marion Woods, Dr Anthony Allworth and Professor David Paterson (Infectious Diseases Unit, Royal Brisbane and Women's Hospital) for assistance during inpatient care and input into manuscript preparation; Dr Graeme Nimmo and Dr Claire Heney (Pathology Queensland) for assistance with susceptibility testing; and Dr Nicholas Kienzle (radiologist) who reviewed the films and approved the descriptions for the figures. Matthew Roberts is supported by a Doctor in Training Research Scholarship from Avant Mutual Group.

Competing interests: No relevant disclosures.

Received 24 Nov 2012, accepted 25 Feb 2013.

1 Carignan A, Roussy J-F, Lapointe V, et al. Increasing risk of infectious complications after transrectal ultrasound-guided prostate biopsies: time to reassess antimicrobial prophylaxis? Eur Urol 2012; 62: 453-459.

2 Loeb S, Carter HB, Berndt SI, et al. Complications after prostate biopsy: data from SEER-Medicare. J Urol 2011; 186: 1830-1834.

3 Bowden FJ, Roberts J, Collignon PJ. Prostate cancer screening and bacteraemia [letter]. Med J Aust 2008; 188: 60.

4 Batura D, Rao GG. The national burden of infections after prostate biopsy in England and Wales: a wake-up call for better prevention. J Antimicrob Chemother 2013; 68: 247-249.

5 Williamson DA, Masters J, Freeman J, Roberts S. Travel-associated extendedspectrum $\beta$-lactamase-producing Escherichia coli bloodstream infection following transrectal ultrasound-guided prostate biopsy [letter]. BJU Int 2012; 109: E21-E22.

6 Hsueh P-R, Hoban DJ, Carmeli Y, et al. Consensus review of the epidemiology and appropriate antimicrobial therapy of complicated urinary tract infections in Asia-Pacific region. J Infect 2011; 63: 114-123.

7 Antibiotic Expert Group. Therapeutic guidelines: antibiotic, version 14. In: eTG complete. Melbourne: Therapeutic Guidelines Limited, 2010. http://www.tg. org.au/index.php?sectionid=71 (accessed Feb 2013).

8 Falagas ME, Giannopoulou KP, Kokolakis GN, Rafailidis PI. Fosfomycin: use beyond urinary tract and gastrointestinal infections. Clin Infect Dis 2008; 46: 1069-1077.

9 Galfano A, Novara G, lafrate M, et al. Prostate biopsy: the transperineal approach. EAU-EBU Update Series 2007; 5: 241-249. doi: 10.1016/ j.eeus.2007.08.001.

10 Abughosh Z, Margolick J, Goldenberg SL, et al. A prospective randomized trial of povidone-iodine prophylactic cleansing of the rectum before transrectal ultrasound guided prostate biopsy. JUrol 2012; Oct 3. pii: S00225347(12)05130-0. doi: 10.1016/j.juro.2012.09.121. [Epub ahead of print.]

11 Taylor AK, Zembower TR, Nadler RB, et al. Targeted antimicrobial prophylaxis using rectal swab cultures in men undergoing transrectal ultrasound guided prostate biopsy is associated with reduced incidence of postoperative infectious complications and cost of care. J Urol 2012; 187: 1275-1279. 